\title{
An Example Concerning Holomorphicity of Meromorphic Mappings Along Real Hypersurfaces
}

\author{
S. IVASHKOVICH \& F. MEYLAN
}

\begin{abstract}
We construct an example of a rational mapping $F$ from $\mathbb{C}^{2}$ to $\mathbb{P}^{2}$ that has indeterminacies on the unit sphere $\mathbb{S}^{3} \subset \mathbb{C}^{2}$ such that $\left.F\right|_{\mathbb{S}^{3}}$ is continuous, the image $K:=F\left(\mathbb{S}^{3}\right)$ is contained in the affine part $\mathbb{C}^{2}$ of $\mathbb{P}^{2}$, and $K$ does not contain any germ of a nonconstant complex curve.
\end{abstract}

\section{Introduction}

Let $F: U \rightarrow \mathbb{P}^{N}$ be a meromorphic mapping from a domain $U \subset \mathbb{C}^{n}$ to the complex projective space. Here we always suppose that $n \geq 2$ and $N \geq 2$. Denote by $I_{F}$ the set of points of indeterminacy of $F$, that is, $z_{0} \in I_{F}$ if and only if $F$ is not holomorphic in any neighborhood of $z_{0}$. As it is well known, $I_{F}$ is an analytic subset of $U$ of codimension at least two. Recall that the full image by $F$ of a point $z_{0}$, denoted as $F\left[z_{0}\right]$, is the set of all cluster points of $F$ at $z_{0}$, that is,

$$
F\left[z_{0}\right]=\left\{x \in \mathbb{P}^{N}: \exists z_{k} \rightarrow z_{0}, z_{k} \notin I_{F} \text { such that } F\left(z_{k}\right) \rightarrow x\right\} .
$$

The mapping $F$ is holomorphic in a neighborhood of $z_{0}$ if and only if $F\left[z_{0}\right]$ is a singleton. Likewise, we can define the full image of $z_{0}$ by $F$ along a closed subset $M$ accumulating to $z_{0}$; for example, $M$ can be a complex curve or a real hypersurface containing $z_{0}$ :

$$
F_{M}\left[z_{0}\right]=\left\{x \in \mathbb{P}^{N}: \exists m_{k} \in M \backslash I_{F}, m_{k} \rightarrow z_{0} \text { such that } F\left(m_{k}\right) \rightarrow x\right\} .
$$

If $M$ is a complex curve, then $F_{M}\left[z_{0}\right]$ is always a singleton, regardless of whether $z_{0}$ is a regular or an indeterminacy point of $F$. As we shall see in our example, the same can happen if $M$ occurs to be a real hypersurface with $z_{0}$ being an indeterminacy point of $F$. The proper image or transform of a closed subset $M \subset$ $U$ under $F$ is defined now to be the union of full images along $M$ of its points. In other words,

$$
F_{M}[M]=\bigcup_{m \in M} F_{M}[m] .
$$

If $M$ is compact, for example, $M=\mathbb{S}^{3} \subset \mathbb{C}^{2}=U$, then

$$
F_{M}[M]=\overline{F\left(M \backslash I_{F}\right)} \text {. }
$$

We denote as $w_{1}, w_{2}$ the standard coordinates in $\mathbb{C}^{2} ; \mathbb{S}^{3}=\left\{\left(w_{1}, w_{2}\right):\left|w_{1}\right|^{2}+\right.$ $\left.\left|w_{2}\right|^{2}=1\right\}$ stands for the unit sphere in $\mathbb{C}^{2}$, and $\mathbb{B}^{2}$ for the open unit ball. Our goal in this note is to construct the following example.

Received February 26, 2014. Revision received January 26, 2015. 
EXAMPLE 1. There exists a rational mapping $F: \mathbb{C}^{2} \rightarrow \mathbb{P}^{2}$ such that:

(i) Its indeterminacy set is $I_{F}=\left\{p_{ \pm}:=(0, \pm 1)\right\}$, its divisor of poles is $P_{F}=$ $\left\{w_{2}= \pm 1\right\}$, and $F_{\mathbb{S}^{3}}\left[p_{ \pm}\right]$are singletons, that is, $\left.F\right|_{\mathbb{S}^{3}}$ is continuous.

(ii) The proper image $K:=F_{\mathbb{S}^{3}}\left[\mathbb{S}^{3}\right]$ of the unit sphere $\mathbb{S}^{3}$ under $F$ does not contain any germ of a nonconstant complex curve.

(iii) In addition, $K$ is contained in an appropriate affine part $W \cong \mathbb{C}^{2}$ of $\mathbb{P}^{2}$.

Let us explain the interest in such an example. It was proved in Lemma 6.6, p. 128 of [P3], and later, independently in [C], that for a germ $M$ of a real analytic hypersurface in $\mathbb{C}^{n}$ and a germ $F$ of a meromorphic mapping near $M$ such that $F_{M}[M] \subset \mathbb{S}^{2 N-1}, 2 \leq n \leq N, F$ is holomorphic near $M$. It is of considerable interest and importance to replace in this result the unit sphere $\mathbb{S}^{2 N-1} \subset \mathbb{C}^{N}$ by an arbitrary compact real analytic set $K \subset \mathbb{C}^{N}$ that does not contain nonconstant germs of complex curves, for instance, smooth and strictly pseudoconvex $K$. This problem has been under discussion among the experts, and our example shows that the before-mentioned conjecture cannot hold if one does not assume that $K$ is smooth. See relevant Remarks 2.1 and 2.2 on these issues at the end of this note.

\section{Construction of the Example}

Step 1. Construction of the mapping. As it is well known, the unit sphere $\mathbb{S}^{3} \subset$ $\mathbb{C}^{2} \subset \mathbb{P}^{2}$, after an appropriate projective transformation of $\mathbb{P}^{2}$ (see (2.3) below) in an appropriate affine chart $U_{0}=\mathbb{C}^{2}$, can be represented in the form

$$
M:=\left\{\operatorname{Im} z_{2}=\left|z_{1}\right|^{2}\right\} .
$$

As a rational map $F$ in question we shall take

$$
F:\left(z_{1}, z_{2}\right) \rightarrow\left(\frac{z_{1}^{3}}{z_{2}}, z_{2}\right) .
$$

For the moment, it is better to consider this map as a mapping from $U_{0}=\mathbb{C}^{2}$ to $\mathbb{P}^{1} \times \mathbb{C}$. Therefore, $F$ is holomorphic on $M \backslash\{0\}$. To see that $F$ is continuous on the whole of $M$, we should remark that $F_{M}[0]=0$, that is, the full image of 0 along $M$ is a singleton. This is nearly obvious because along $M$ we have $\left|z_{2}\right| \geq\left|z_{1}\right|^{2}$, and therefore along $M$ the first function in (2.2) is $O\left(\left|z_{1}\right|\right)$.

Set $l:=M \cap\left\{z_{1}=0\right\}$, which is a real line. We easily check that $F$ is the identity when restricted to $l$ and that it is locally injective on $M \backslash l$. The latter can be seen by computing the Jacobian of $F$, and therefore $\left.F\right|_{M \backslash l}: M \backslash l \rightarrow$ $F(M \backslash l)$ is a three-to-one cover. This means that $F(M \backslash l)$ is a strictly pseudconvex hypersurface. Therefore, it cannot contain any germ of a complex curve. As a result, a germ of a complex curve in $F(M)$ should be contained in $F(l)=l$, which is impossible. Thus, $F(M)$ does not contain germs of complex curves.

Step 2. Globalization. It is easy to check that the projective transformation

$$
R=\left\{\begin{array}{l}
z_{1}=\frac{w_{1}}{1-w_{2}}, \\
z_{2}=i \frac{1+w_{2}}{1-w_{2}}
\end{array}\right.
$$


sends the unit sphere $\mathbb{S}^{3}=\left\{\left|w_{1}\right|^{2}+\left|w_{2}\right|^{2}=1\right\}$ to our model $M$. Writing $F$ as a mapping to $\mathbb{P}^{2}$, that is, as

$$
F:\left(z_{1}, z_{2}\right) \rightarrow\left[z_{1}^{3}: z_{2}^{2}: z_{2}\right]
$$

and composing it with $R$, we get the following expression of our map in coordinates $w_{1}, w_{2}$ :

$$
F:\left(w_{1}, w_{2}\right) \rightarrow\left[w_{1}^{3}:-\left(1+w_{2}\right)^{2}\left(1-w_{2}\right): i\left(1+w_{2}\right)\left(1-w_{2}\right)^{2}\right] .
$$

We refer to (2.5) as to the form of the mapping $F$ in coordinates $\left(w_{1}, w_{2}\right)$. This is the mapping of our example. Now let us prove the assertions (i)-(iii) as they are stated in the Introduction.

(i) It is easy to see that it has two points of indeterminacy $p_{ \pm}=(0, \pm 1)$ and that it behaves at these points similarly. The behavior at $p_{-}$was already studied, and the behavior at $p_{+}$is the same. The divisor of poles is $\left\{w_{2}= \pm 1\right\}$, as required.

(ii) $K:=F\left(\mathbb{S}^{2}\right)$ cannot contain any germ of a nonconstant complex curve. Indeed, let $C \subset K$ be such a germ. Then $C$ should intersect an affine neighborhood either of $p_{+}$or of $p_{-}$, and this was proved to be not the case.

(iii) To prove that $K$ is situated in an appropriate affine part of $\mathbb{P}^{2}$, consider in homogeneous coordinates $\left[\zeta_{0}: \zeta_{1}: \zeta_{2}\right]$ the line

$$
L:=\left\{\zeta_{2}-i \zeta_{1}=0\right\}
$$

Putting $F(w)$ into this equation, we get

$$
\zeta_{2}-i \zeta_{1}=i\left(1+w_{2}\right)\left(1-w_{2}\right)^{2}+i\left(1+w_{2}\right)^{2}\left(1-w_{2}\right)=2 i\left(1-w_{2}^{2}\right)
$$

The latter is not zero on $\mathbb{S}^{3}$ if and only if $\mathrm{w}_{2} \neq \pm 1$, that is, if $w$ is not $p_{ \pm}$. But at $p_{-}$ along $\mathbb{S}^{3}$ our map takes the value $[0: 0: 1] \notin L$ and at $p_{+}$the value $[0: 1: 0] \notin L$. Therefore, $F_{\mathbb{S}^{3}}\left[\mathbb{S}^{3}\right] \subset \mathbb{P}^{2} \backslash L=: W \cong \mathbb{C}^{2}$. Now we can make the coordinate change

$$
\left\{\begin{array}{l}
\xi_{0}=\zeta_{0}, \\
\xi_{1}=\zeta_{1}, \\
\xi_{2}=\zeta_{2}-i \zeta_{1}
\end{array}\right.
$$

in $\mathbb{P}^{2}$ and write our map in the form

$$
F:\left(w_{1}, w_{2}\right) \rightarrow\left[w_{1}^{3}:-\left(1+w_{2}\right)\left(1-w_{2}^{2}\right): 2 i\left(1-w_{2}^{2}\right)\right]
$$

or, in the affine charts,

$$
F:\left(w_{1}, w_{2}\right) \rightarrow\left(-\frac{i}{2} \frac{w_{1}^{3}}{1-w_{2}^{2}}, \frac{i}{2}\left(1+w_{2}\right)\right) .
$$

This is the final form of $F$ as a mapping from $\mathbb{C}^{2} \subset \mathbb{P}^{2}$ to $\mathbb{C}^{2} \subset \mathbb{P}^{2}$, that is, $K \subset \mathbb{C}^{2}$ if $F$ is given in the form (2.10).

Let us make a few additional remarks about our example. 


\section{REMARK 2.1.}

1. The image $K=F\left(\mathbb{S}^{2}\right)$ in our example is not smooth. Indeed, it is given by the equation

$$
\left(\operatorname{Im} z_{2}\right)^{3}=\left|z_{1} z_{2}\right|^{2} \text {. }
$$

It is easy to see that the set of singular points of (2.11) is exactly our line $l=F(M) \cap\left\{z_{1}=0\right\}$ considered before. Outside of $l$, our $K$ is a spherical hypersurface.

2. The nonsmoothness of $K$ can be overcome at the cost of increasing the dimension of the image. Our example written as a map $\mathbb{C}^{2} \rightarrow \mathbb{C}^{3}$, that is, as

$$
F:\left(z_{1}, z_{2}\right) \rightarrow\left(\frac{z_{1}^{3}}{z_{2}}, z_{2}, 0\right),
$$

takes its values in the smooth algebraic hypersurface

$$
K=\left\{y_{3}+y_{2}^{3}-\left|z_{1} z_{2}\right|^{2}=0\right\} .
$$

However, this hypersurface contains the complex curve $C=\left\{z_{2}=z_{3}=0\right\}$.

REMARK 2.2. In equal dimensions with strictly pseudoconvex both $M$ and $K$, such an example does not exist. Namely, the following is true.

Proposition 2.1. Let $(M, 0)$ be a germ of strictly pseudoconvex hypersurface in $\mathbb{C}^{n}, n \geq 2$, and let $K$ be a compact strictly pseudoconvex hypersurface in $\mathbb{C}^{n}$. Let $F: V \rightarrow \mathbb{C}^{n}$ be a meromorphic mapping from some neighborhood of the origin $V \subset \mathbb{C}^{n}$ to $\mathbb{C}^{n}$ such that $F(M) \subset K$. Then $F$ is holomorphic in a neighborhood of the origin.

Proof. If $F$ is constant, then there is nothing to prove. If not, then it is locally biholomorphic at every point of $M \backslash I_{F}$. Now we have two cases.

Case 1. $M$ is spherical. Then $K$ is spherical as well. Set $p:=F(0)$ and let $j:(K, p) \rightarrow\left(\mathbb{S}^{2 n-1}, 0\right)$ be a germ of a biholomorphic mapping. By the result of [P1] germ $j$ extends locally biholomorphically along any path in $K$. Take some point $a \in M \backslash I_{F}$ and set $b:=F(a)$. Take a path $\gamma$ in $M$ from 0 and $a$. Set $\beta:=F(\gamma)$; it is a path from $p$ to $b$ in $K$. The composition $j \circ F$ extends along $\gamma^{-1}$ locally biholomorphically from $a$ to 0 by the mentioned result of [P1]. Since $j$ extends along $\beta$ and therefore $j^{-1}$ extends along $j\left(\beta^{-1}\right)$, we can take the composition $j^{-1} \circ(j \circ F)$ and extend it from $a$ to 0 to obtain the holomorphicity of $F$ at the origin.

Case 2. $M$ is not spherical. Then $K$ is not spherical as well. We can then apply another theorem of Pinchuk [P2]. Take any Moser chain through 0 on $M$ that is not contained entirely in $M \cap I_{F}$, say a path $\gamma:[0,1] \rightarrow M$ such that $\gamma(0) \notin I_{F}$ and $\gamma(1)=0$. Extend $F$ along $\gamma$ by [P2] to conclude that $F$ is holomorphic at zero.

Acknowledgments. We are grateful to Rasul Shafikov and Alexander Sukhov for stimulating discussions, in particular, for Remark 2.1 about our example and for giving us the possibility to consult the thesis of Pinchuk [P3]. 


\section{References}

[C] S. Chiappari, Holomorphic extension of proper meromorphic mappings, Michigan Math. J. 38 (1991), 167-174.

[P1] S. Pinchuk, On the analytic continuation of holomorphic mappings, Math. USSR Sb. 27 (1975), no. 3, 375-392.

[P2] On holomorphic mappings of real-analytic hypersurfaces, Math. USSR Sb. 34 (1978), no. 4, 503-519.

[P3] Analytic continuation of holomorphic mappings and the problem of holomorphic classification of multidimensional domains, Doctor thesis (Habilitation), Chelyabinsk, 1979.

S. Ivashkovich

UFR de Mathématiques

Université de Lille-1

59655 Villeneuve d'Ascq

France

ivachkov@math.univ-lille1.fr

\section{IAPMM}

Nat. Acad. Sci. Ukraine

Naukova 3b, Lviv 79601

Ukraine
F. Meylan

Département de Mathématiques

Université de Fribourg

1700 Fribourg

Suisse

francine.meylan@unifr.ch 